\title{
SHORT REPORT \\ Did AIDS mortality decrease the number of lifetime sexual partners in Kenya: an ecological analysis?
}

\author{
C. R. KENYON ${ }^{1,2 *}$, I. SCHWARTZ ${ }^{3}$ AND J. BUYZE ${ }^{1}$ \\ ${ }^{1}$ HIVISTI Unit, Institute of Tropical Medicine, Antwerp, Belgium \\ ${ }^{2}$ Division of Infectious Diseases and HIV Medicine, University of Cape Town, South Africa \\ ${ }^{3}$ Department of Infectious Diseases, University of Antwerp, Belgium
}

Received 15 April 2015; Final revision 19 June 2015; Accepted 26 June 2015;

first published online 10 July 2015

\section{SUMMARY}

We assessed if there has been a decline in the median number of reported lifetime sexual partners in Kenya following the AIDS epidemic. The Wilcoxon rank-sum test was used to compare the median and interquartile range (IQR) of the number of lifetime sexual partners for men aged 20-54 years in the 1993 and 2008 Kenyan Demographic Health Surveys. The median number of sexual partners in 1993 increased rapidly to 10 partners reported at age 30 years then plateaued at this level. In 2008, the median number of sexual partners plateaued at around half the value of the 1993 plateau. The median number of lifetime sexual partners for men aged 20-54 years declined from 10 (IQR 4-20) in 1993 to 3 (IQR 2-7) in $2008(P<0 \cdot 001)$. This decline could be due to a combination of the effects of AIDS mortality and a misreporting bias.

Key words: AIDS, epidemiology, sexually transmitted infections (STIs).

It is important to correctly identify the factors responsible for the decline of sexually transmitted infection (STI) rates in much of Southern and Eastern Africa. A prevalent view is that behaviour change in the form of adopting various safer sex practices in combination with better treatment of STIs was responsible [1]. Studies from men who have sex with men (MSM) in the USA have, however, provided evidence that AIDS mortality played a significant role [2, 3].

The effect of AIDS mortality could have been mediated via three pathways: (i) individuals most at risk of HIV acquisition and transmission were removed from the population, (ii) people living in areas with higher AIDS mortality may have been more likely to change sexual behaviour [3], and (iii)

\footnotetext{
* Author for correspondence: Professor C. R. Kenyon, HIV/STI Unit, Institute of Tropical Medicine, Antwerp, Belgium. (Email: ckenyon@itg.be)
}

depletion of the pool of high-risk persons might have made it difficult for the remaining high-risk persons to find sufficient partners with whom to engage in risky sex [2].

Since peak AIDS mortality rates in some Southern and Eastern African countries were higher than that those observed in MSM in the USA, it is plausible that AIDS mortality may have played a significant role in reducing HIV/STI transmission in these countries [3-5]. The countries with generalized HIV epidemics that have experienced large declines in STI incidence include Botswana, Kenya, Lesotho, Malawi, Mozambique, South Africa, Tanzania, Uganda, Zambia and Zimbabwe $[1,5]$.

The effect of AIDS mortality on the structure of sexual networks and, hence, STI transmission is difficult to directly assess outside of longitudinal cohort studies that regularly collect sexual behaviour data and HIV serostatus. One indirect way to assess this effect is to see if high AIDS mortality in a population is 
followed by a decline in the lifetime number of sexual partners in that population. The rationale is that persons with the most sexual partners would be more likely to become HIV infected and, therefore, die, thus lowering the mean and median lifetime number of partners in that population. The only datasets we could find that allowed us to test this hypothesis were the 1993 and 2008 Demographic and Health Surveys (DHS) from Kenya. Both surveys asked men and women about their lifetime number of sexual partners and both used a similar two-stage stratified sampling approach that, once weighted, provided prevalence estimates that were nationally representative (excluding the North-eastern province, which was not sampled in the 1993 survey). The 1993 survey included men aged 20-54 years and the 2008 survey men aged 15-54 years. Because of these differences in sampling, we restricted our comparisons to men aged 20-54 years living outside the North-eastern province $(n=2336$ in 1993, $n=2541$ in 2008). Women aged 15-49 years reported a median of two lifetime sexual partners in 1993 and one lifetime sexual partner in 2008 and a slight decline in the mean number of sexual partners from 2.2 in 1993 to 1.9 in 2008. However, DHS surveys have been shown to be particularly susceptible to underreporting the number of sexual partners by women $[6,7]$. As a result, only the results for men are presented.

The estimated number of deaths per year due to AIDS in Kenya increased from $<5000$ in 1990 to peak at 150000 in 2003 and declined to 85000 in 2009 [8, 9]. The 1993 and 2008 surveys therefore provided us with estimates of lifetime sexual partners in the pre- and post-peak AIDS eras, respectively. Since there were 15 years between the surveys, the men aged 20-39 years in the 1993 survey would have become $35-54$ years by 2008 . We compared the median and interquartile range (IQR) of the number of lifetime sexual partners, first for men aged 20-54 years in 1993 with those in 2008 and, second, for men aged 20-39 years in 1993 with those aged 3554 years in 2008. Wilcoxon rank-sum test was used for this analysis. The analyses include all men in these age groups regardless of whether they reported having had sex before. The analyses were performed using R v. 3.1.0 (R Foundation, Austria). All analyses were adjusted to account for the complex sampling strategies of the surveys.

As shown in Figure 1, the median number of sexual partners by age in 1993 increased rapidly to 10 partners reported at age 30 years then plateaued at this level. In 2008, the median number of sexual partners plateaued at around half the value of the 1993 plateau. The median [interquartile range (IQR)] number of lifetime sexual partners for men aged 20-54 years declined from 10 (IQR 4-20) in 1993 to 3 (IQR 2-7) in $2008(P<0 \cdot 001)$. The median number of lifetime sexual partners declined from 10 (IQR 4-20) in men aged 20-39 years in 1993 to 4 (IQR 2-8) in men aged 35-54 years in $2008(P<0 \cdot 001)$. Comparisons of the histograms of lifetime sexual partners of 20to 39 -year-olds in 1993 with 35 - to 54-year-olds in 2008 revealed a $55 \%$ decline in the proportion of men reporting $\geqslant 10$ sexual partnerships (from $50 \cdot 2 \%$ to $22 \cdot 8 \%$, respectively; data not shown). There was a similar difference between the two surveys when all 20- to 54-year-olds were compared.

How can we explain the decline in the reported number of lifetime sexual partners in men aged 20 39 years in 1993 with this same age group of men 15 years later? This decline could be explained by differences in the questionnaire design, differences in sampling, misreporting bias in 1993/2008 or mortality due to AIDS or other causes. The first explanation can be discarded, as both the lifetime sexual partners question and the preceding framing questions were almost identical in both surveys. The second explanation is less likely, given that our analysis accounted for the slight differences in survey design. It is possible that in the 15-year period between the two samples, reporting a greater number of lifetime partners became less socially acceptable and thus men became more likely to deflate (or less likely to inflate) their reported number of partners. We are unable to discount this possibility, but it would not easily explain the greater decline in lifetime partners in ethnic groups most affected by HIV. HIV prevalence in Kenya's ethnic groups varies between $0 \cdot 8 \%$ and $20.8 \%$ and the drop in lifetime sexual partners between 1993 and 2008 for both men and women was greatest in the two ethnic groups with the highest HIV prevalences (Luo and Luhya, see [10]). We cannot, however, exclude the possibility that the ethnic groups most affected by HIV were most likely (due to a social desirability bias) to underreport lifetime partners. Despite these reservations, in our estimation AIDS mortality provides a better explanation for the decline in reported lifetime partners. It also fits with the timing of the HIV epidemic and is commensurate with the evidence of the effect of AIDS mortality on HIV/STI incidence in MSM in USA. In addition, it is congruent with modelling and ecological evidence that suggests that 


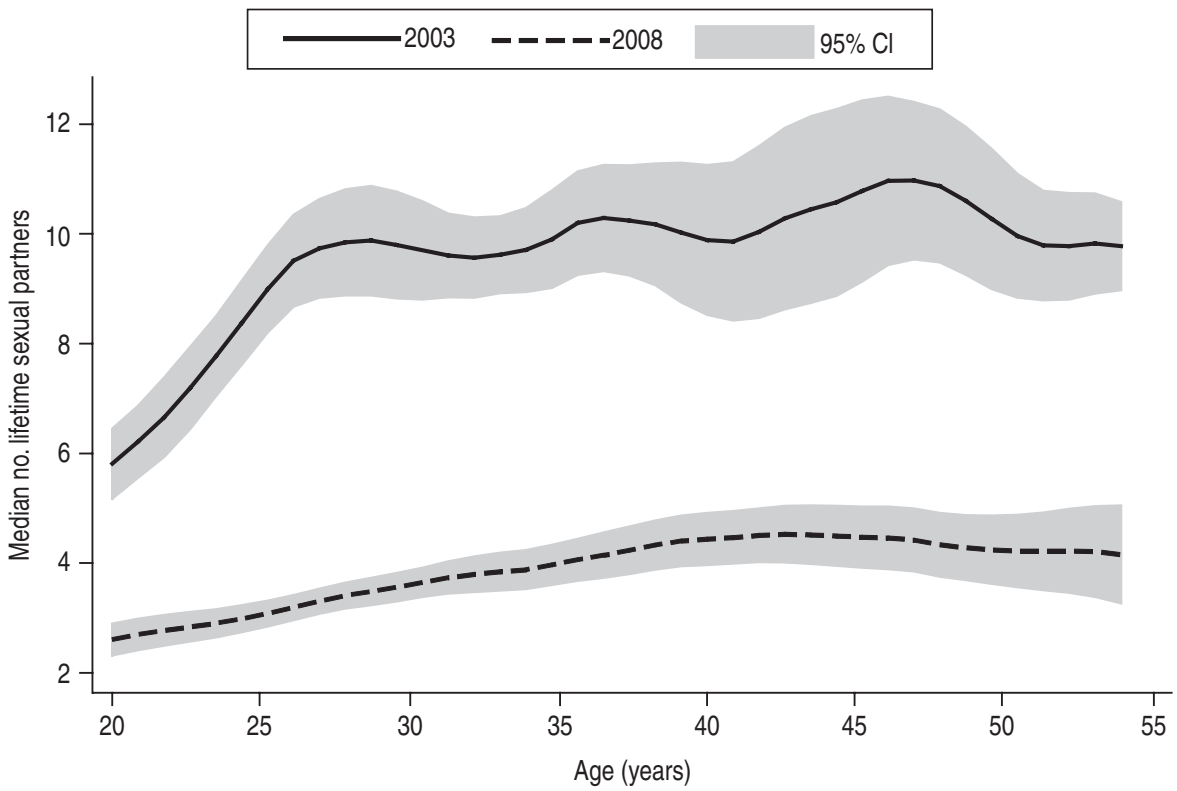

Fig. 1. The median number of lifetime sexual partners reported by men in 1993 and 2008 (from Kenya Demographic and Health Surveys 1998 and 2003). The shaded grey area represents the $95 \%$ confidence interval (CI).

AIDS mortality played a significant role in the decline of the prevalence of syphilis, male genital ulcers and male urethral discharge in Southern and Eastern Africa $[1,5,11]$. Furthermore, a study from an open cohort in Masaka, Uganda found that $25 \%$ of both men and women reported fewer lifetime partners in a later survey (2007) compared to the earlier survey (2000) [12]. The authors of this paper did not quantify the decline in lifetime partners but speculated that this could be due to misreporting or AIDS mortality. Unfortunately, we could not test the relationship between lifetime sexual partners and AIDS mortality elsewhere as we could not find another country that collected DHS data on lifetime sexual partners in both the pre- and post-AIDS periods. Investigating this relationship in demographic and surveillance sites that have collected information on lifetime partners would constitute useful future research. It should be emphasized that we do not have any direct evidence to substantiate the differential AIDS mortality explanation. Furthermore, the magnitude of the decline in sexual partners may be too large to be explained solely by AIDS mortality. Other factors, such as misreporting bias, may have played a role.

The parallels with the HIV epidemic in MSM in the West may be relevant. AIDS mortality led to declines in HIV/STI incidence both directly through the effect of removing more central players from sexual transmission networks and indirectly through behaviour change $[2,3]$. Antiretroviral therapy (ART) has, to some extent, negated the effect of both these mechanisms and thereby likely played a role in the re-emergence of HIV/STIs in this group [13]. If AIDS mortality played a significant role in the decline of AIDS and other STIs in Southern and Eastern Africa then this has important implications. One is that behaviour change may not have been as extensive as is commonly believed. The widespread availability of ART could then lead to a rebound in HIV/STI incidence. The recent increases in reported multiple partnering in Uganda and South Africa and the increase in HIV incidence in Uganda suggest that this process may have already begun [14, 15]. Campaigns to effect more profound behaviour change may be warranted.

\section{ACKNOWLEDGEMENTS}

No funding was received for this study.

\section{DECLARATION OF INTEREST}

None.

\section{REFERENCES}

1. Johnson LF, et al. The effect of syndromic management interventions on the prevalence of sexually transmitted infections in South Africa. Sexual \& Reproductive Healthcare 2011; 2: 13-20.

2. Boily MC, et al. The impact of the transmission dynamics of the HIV/AIDS epidemic on sexual behaviour: a 
new hypothesis to explain recent increases in risk-taking behaviour among men who have sex with men. Medical Hypotheses 2005; 65: 215-226.

3. Chesson HW, Dee TS, Aral SO. AIDS mortality may have contributed to the decline in syphilis rates in the United States in the 1990s. Sexually Transmitted Diseases 2003; 30: 419-424.

4. Hosegood V, Vanneste AM, Timaeus IM. Levels and causes of adult mortality in rural South Africa: the impact of AIDS. AIDS 2004; 18: 663-671.

5. Kenyon CR, Osbak K, Chico RM. What underpins the decline in syphilis in Southern and Eastern Africa? An exploratory ecological analysis. International Journal of Infectious Diseases 2014; 29: 54-61.

6. Beauclair R, et al. Evaluating audio computer assisted self-interviews in urban south African communities: evidence for good suitability and reduced social desirability bias of a cross-sectional survey on sexual behaviour. BMC Medical Research Methodology 2013; 13: 11.

7. Morris M, et al. Comparing estimates of multiple and concurrent partnerships across population based surveys: implications for combination HIV prevention. AIDS and Behavior 2013.

8. UNAIDS/WHO. UNAIDS/WHO epidemiological fact sheet - 2004 update. Geneva, 2005.
9. National AIDS and STI Control Programme (NASCOP) Kenya. Kenya AIDS Response Progress Report. Nairobi: NASCOP, 2014.

10. Kenyon $\mathbf{C}$, et al. Male circumcision and sexual risk behaviors may contribute to considerable ethnic disparities in HIV prevalence in Kenya: an ecological analysis. PLoS ONE 2014; 9: e106230.

11. Kenyon $\mathbf{C}$, et al. The changing relationship between bacterial STIs and HIV prevalence in South Africa - an ecological study. International Journal of STD and AIDS 2015; 26: 556-564.

12. Todd $\mathbf{J}$, et al. Reported number of sexual partners: comparison of data from four African longitudinal studies. Sexually Transmitted Infections 2009; 85 (Suppl. 1): i72-80.

13. Chesson HW, Gift TL. Decreases in AIDS mortality and increases in primary and secondary syphilis in men who have sex with men in the United States. Journal of Acquired Immune Deficiency Syndromes 2008; 47: 263-264.

14. Shisana O, et al. Onoya D. South African National HIV Prevalence, Incidence and Behaviour Survey, 2012. Cape Town: HSRC Press, 2014.

15. Uganda Ministry of Health. Uganda AIDS indicator survey 2011. Ministry of Health, and Calverton, Maryland, ICF Kampala, Uganda, 2012. 